\title{
L'émergence du marché de la compensation des zones humides aux États-Unis : impacts sur les modes d'organisation et les caractéristiques des transactions
}

\section{The emergence of mitigation banking in the United States: impacts on the modes of organization and the characteristics of transactions}

Pierre Scemama and Harold Levrel

IFREMER, UMR M101, AMURE, Unite Econ Maritime, F-29280 Plouzane, France.

*: Corresponding authors : Pierre Scemama, email address : pierre.scemama@ifremer.fr ; Harold Levrel, email address : harold.levrel@ifremer.fr

\section{Résumé :}

Aux États-Unis, le Clean Water Act requiert des mesures compensatoires pour les écosystèmes aquatiques qui seraient endommagés par des projets d'aménagement. A partir des années 90 , pour améliorer la qualité du système, on a assisté à l'émergence d'un système marchand pour encadrer les transactions liées à la mise en place des actions de compensation. Le cadre théorique de l'économie néo-institutionnel stipule que le choix du mode d'organisation d'une transaction se fait sur la base de la minimisation des coûts de transaction. Ces coûts sont liés aux dispositifs de coordination qui sont propres à chaque mode d'organisation et dont l'efficacité - en termes de coûts de transaction dépend des caractéristiques de la transaction : la spécificité des actifs, l'incertitude et la fréquence des transactions.

Les transactions liées à la compensation des impacts impliquent un investissement important dans du capital naturel - l'écosystème aquatique - de manière à produire un gain environnemental équivalent en qualité et en quantité aux pertes liées au dommage. L'application de la théorie néo-institutionnelle à l'étude de ces transactions permet de montrer que ces transactions ont des caractéristiques originales qui nécessitent des modes d'organisation de formes hybrides. La volonté d'inscrire la compensation dans un système marchand implique un alignement des caractéristiques des transactions avec un risque de diminution de la spécificité du capital naturel. Par conséquent il est nécessaire de maintenir un système de régulation fort pour garantir la qualité de la compensation.

Mots clés: Économie Néo-Institutionnelle; mode d'organisation; compensation écologique ; banques de compensation 


\begin{abstract}
:
In the United States, the Clean Water Act requires compensatory measures for aquatic ecosystems damaged by development projects. From the 90's to improve the quality of the system, we have seen the emergence of a market system to supervise the transactions related to the implementation of actions for compensation. The theoretical framework of neo-institutional economics states that the choice of the organization of a transaction is based on the minimization of transaction costs. These costs are related to the coordination mechanisms that are specific to each type of organization and which efficiency - in terms of transaction costs - depends on the characteristics of the transaction : asset specificity, uncertainty and frequency of the transactions.

Transactions related to compensation involve a significant investment in natural capital - the aquatic ecosystem - to produce an environmental gain equivalent in quality and quantity, to the losses related to the damage. The application of the neo-institutional theory to the study of these transactions allows us to show that these transactions have original characteristics that require organizational modes of hybrid forms. The desire to include the compensation in a trading system involves an alignment of the characteristics of transactions with a risk reduction of the specificity of natural capital. Therefore it is necessary to maintain a strong regulatory system to ensure the quality of the compensation.
\end{abstract}

Keywords: New Institutional Economics ; organizational structure ; ecological compensation ; mitigation banking 


\section{INTRODUCTION}

Le bien-être social des populations à une période donnée est fonction de l'utilité dégagée par la consommation des biens et services disponibles à cette période (Ramsey [1928]). La consommation future et donc le bien-être social sont fonction des stocks actuels de capitaux (Mäler et al. [2008]). II existe différents types de capitaux impliqués dans les fonctions de production: le capital manufacturé (actifs immobiliers, matériels de production et produits finis), le capital humain (tout ce qui contribue à la force de travail dégagée par l'homme comme l'éducation ou la santé), le capital social (qui comprend l'ensemble des réseaux sociaux facilitant l'efficacité organisationnelle) et le capital naturel.

Le capital naturel peut être divisé en quatre catégories (Clewell and Aronson, [2010]): le capital naturel renouvelable (les espèces vivantes et les écosystèmes), le capital naturel réapprovisionnable (la couche d'ozone, l'eau potable, etc.), le capital naturel cultivé (les cultures, les plantations forestières, etc.) et le capital naturel non renouvelable (le pétrole, les minerais, etc.).

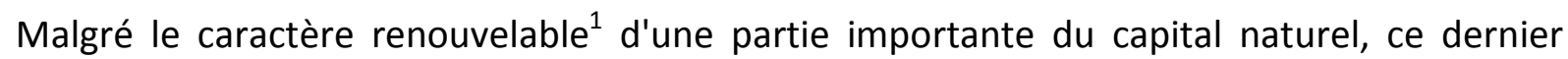
subit une forte érosion pour deux raisons (UICN [2009] ; Butchart et al. [2010] ; Hoffmann et al. [2010]). La première est l'augmentation des activités humaines qui dégradent les écosystèmes à travers la surexploitation, l'introduction d'espèces invasives, la destruction et la pollution des habitats ou le réchauffement climatique (Sala et al. [2000] ; Balmford et Bond [2005]). La seconde est le manque d'investissement dans le capital naturel qui permet la fourniture des services écosystémiques ${ }^{2}$. Ce constat entraîne l'augmentation d'initiatives visant à encourager l'investissement dans le capital naturel comme par exemple les engagements de la CDB qui vise la restauration d'au moins $15 \%$ des écosystèmes dégradés d'ici 2020 (CDB [2011]).

Parmi le capital naturel concerné on trouve les écosystèmes aquatiques dont la moitié de leurs surfaces auraient disparu depuis 1900 (Russi et al. [2012]). Ce constat de la dégradation des écosystèmes aquatiques conduit à des innovations institutionnelles visant à réduire leur

\footnotetext{
${ }^{1}$ Le capital renouvelable réapprovisionnable et cultivé peut être exploité de façon à respecter les taux de renouvellement, alors que le capital naturel de type non renouvelable est un stock de biens dont l'exploitation conduit à la liquidation d'une partie du stock (Rees [1995]).

${ }^{2}$ Le Millenium Ecosystem Assessment identifie quatre catégories de services écosystèmiques (MEA [2005]): les services d'approvisionnement qui renvoient aux ressources naturelles directement exploitées; les services de régulation qui représentent les fonctions écosystémiques telles que la purification de l'eau ou le recyclage des déchets; les services de support qui sont les processus élémentaires nécessaires à la reproduction du vivant sur la Terre ; les services culturels qui sont liés aux activités récréatives en lien avec la nature et au sentiment de bien-être de manière générale.
} 
destruction et à encourager leur restauration. La mise en place de la Section 404 du Clean Water Act (CWA) américain représente l'une de ces innovations institutionnelles. Elle a pour objet de limiter les droits d'usage des propriétaires des zones humides en vue de mieux préserver ces écosystèmes. Ainsi un propriétaire qui souhaiterait utiliser sa zone humide pour y construire une route ou un bâtiment doit acquérir un droit de détruire qui est délivré par I'USACE sous condition de respecter une procédure nommée mitigation sequence ${ }^{3}$. Le renforcement de la régulation des usages sur les zones humides fait apparaître une nouvelle transaction liée à la mise en place de compensations environnementales, correspondant à la troisième étape de la mitigation sequence.

Pour étudier les caractéristiques de cette transaction, il est possible d'avoir recours à différents cadres théoriques. Nous proposons, dans ce papier, d'utiliser l'approche de la théorie néo-institutionnelle. D'après cette théorie, toute transaction requiert un mode d'organisation caractérisé par des dispositifs spécifiques de coordination qui génèrent des coûts de transaction, variant selon les caractéristiques de la transaction. Toujours d'après cette théorie, le choix du mode organisationnel s'opère de manière à aligner les caractéristiques de la transaction et le mode d'organisation dans l'optique de minimiser les coûts de transaction, c'est-à-dire les coûts de détermination du juste prix, les coûts de négociation et de contractualisation (Williamson [1985] ; Ménard et Shirley [2008] ; Annexe 1).

Dans le cadre des compensations environnementales, cette transaction implique un investissement dans du capital naturel que représente un écosystème aquatique. Ce sont les caractéristiques de cet investissement qui déterminent la quantité et la qualité des gains environnementaux qui sont produits.

Le recours à l'économie néo-institutionnelle est en expansion dans l'étude des politiques environnementales (Coggan et al. [2010] ; McCann [2013] ; Pannell et al. [2013]). Ces publications étudient généralement les coûts de transaction sous deux angles: une approche théorique visant à faire un lien entre le cadre des coûts de transaction et celui de l'analyse des politiques environnementales (McCann et al. [2005] ; Coggan et al. [2013] ; Marshal [2013]) ; l'évaluation des coûts de transactions associés à la mise en œuvre de politiques environnementales (Falconer et al. [2001] ; Rorstad et al. [2007] ; Steele [2009] ; Nilsson [2009] ; Mettepenningen et al. [2011]). En revanche, l'analyse des caractéristiques des transactions - spécificité des actifs, incertitudes, fréquence des transactions -, qui peuvent expliquer comment les niveaux coûts de transaction varient selon différentes

\footnotetext{
${ }^{3}$ II s'agit d'une procédure en trois étapes définies par la Section 404 du CWA. La première étape est "l'évitement " qui implique que tous les impacts négatifs sur les ressources aquatiques doivent être évités s'il existe au moins une alternative ayant moins d'impacts négatifs. La deuxième étape est la " réduction » qui nécessite que tous les impacts négatifs qui n'ont pas pu être évités doivent être minimisés. La dernière étape est "la compensation" qui implique que tous les impacts résiduels, suite à l'application des étapes d'évitement et de réduction, doivent être compensés. Le montant et la qualité de la compensation ne peuvent en aucun cas permettre à la compensation de se substituer aux étapes d'évitement et de réduction.
} 
modalités de mises en œuvre d'une politique environnementale, ne semble pas avoir été développée dans la littérature.

L'hypothèse que nous souhaitons discuter dans cet article est que ces transactions, qui mettent en jeu des investissements dans du capital naturel, ont des caractéristiques particulières qui pèsent sur le choix du mode d'organisation, sur l'efficacité des changements institutionnels et in fine sur la qualité de la compensation environnementale.

Pour tester cette hypothèse, nous détaillerons, dans une première partie, comment la compensation des impacts autorisés sur les zones humides est mise en œuvre aujourd'hui, en détaillant les caractéristiques des transactions associées, les coûts de transactions générés par celles-ci, et leurs conséquences sur l'efficacité du système de régulation. Dans une seconde partie nous étudierons comment l'émergence d'une nouvelle forme de gouvernance au cours des années 2000 - les banques de compensation - a représenté une réponse organisationnelle à l'échec du système réglementaire originel, et en quoi elles ont pu créer une plus grande efficacité de la politique environnementale autour des zones humides. Nous discuterons ensuite les enseignements tirés de notre analyse au regard de notre hypothèse de départ.

\section{La compensation des impacts autorisés sur les zones humides}

\section{A. La compensation et la transaction qui en découle}

Aux Etats-Unis, la section 404 du CWA a créé un système d'autorisation conditionnelle des impacts: si un aménageur désire détruire un écosystème aquatique, il doit obtenir un permis qui est délivré par l'agence de I'US Army Corps of Engineers (USACE) du district concerné, après consultation du bureau régional de I'US Environmental Protection Agency (EPA). II peut choisir d'autoriser l'impact, de l'interdire ou de l'autoriser à condition que l'aménageur "crée" une certaine quantité d'écosystème aquatique en compensation de la perte générée par l'aménagement.

L'unité d'analyse de l'économie néo-institutionnelle est la transaction, ainsi en suivant la définition retenue par la théorie $e^{4}$ nous nous intéresserons à l'étude de la transaction qui unit (Figure 1):

- le développeur qui a besoin d'un gain environnemental équivalent au dommage dont il est à l'origine ;

- le professionnel du génie écologique qui investit dans le capital naturel que représente la zone humide, à travers des actions de compensation, de façon à répondre à la demande du développeur ;

- le régulateur qui contrôle l'équivalence entre les pertes et les gains de capital naturel.

\footnotetext{
4 "Une transaction a lieu lorsqu'un bien ou un service est transféré à travers une interface technologiquement séparable. Une étape d'activité se termine et une autre commence" (Williamson [1985], p1)
} 


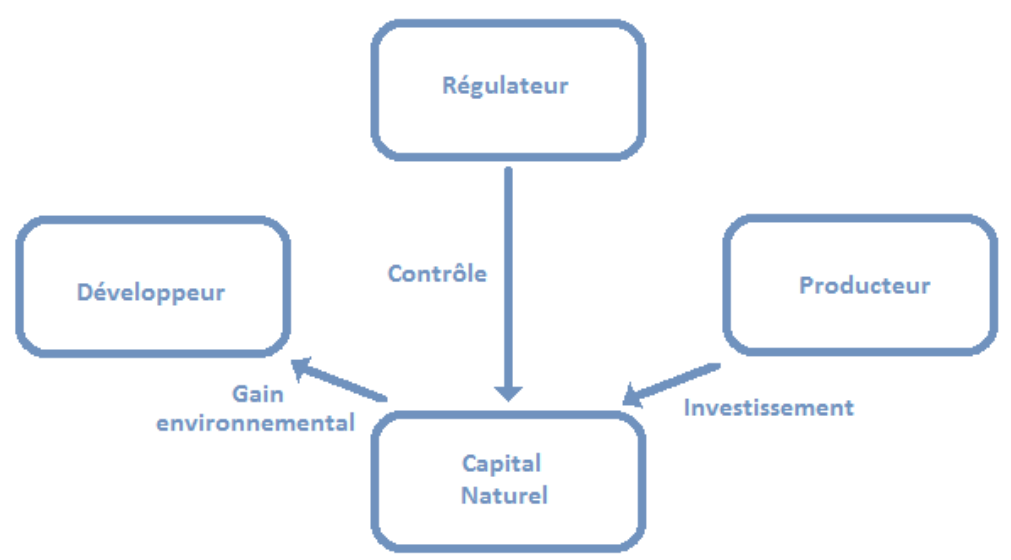

Figure 1 - Représentation de la transaction que nous étudions dans le cadre de la compensation écologique. II s'agit d'une représentation simple de la transaction destinée à faire apparaître les acteurs protagonistes (les deux parties et la partie tiers qui la régule) et leur rôle vis à vis du capital naturel au cœur de la transaction.

Au regard de ce que nous avons mentionné plus haut, le professionnel du génie écologique déploie un investissement dans un outil de production - l'écosystème aquatique correspondant au capital naturel. Ce sont les caractéristiques de cet investissement qui déterminent la quantité et la qualité du gain environnemental correspondant au produit vendu au développeur. C'est pourquoi il semble important d'étudier la spécificité des actifs à mobiliser, l'incertitude qui pèse sur les transactions et la fréquence des transactions.

\section{B. La spécificité des actifs déployés dans ces transactions}

Un actif spécifique est défini comme un investissement durable et spécialisé qui ne peut pas être redéployé vers un usage alternatif sans entraîner de perte de sa valeur productive. II existe plusieurs types de spécificité qui peuvent être associées aux actifs naturels que représentent les écosystèmes: spécificité physique, spécificité de site, spécificité dédiée, spécificité de savoir-faire et spécificité de marque (Tableau 1). Nous proposons de détailler leur contenu et leur influence sur les coûts de transaction à partir de l'exemple des écosystèmes aquatiques.

\begin{tabular}{|c|c|c|}
\hline \multicolumn{3}{|r|}{ Spécificité des actifs déployés } \\
\hline \multirow{3}{*}{$\frac{\text { Spécificité }}{\frac{\text { du capital }}{\text { naturel }}}$} & $\begin{array}{l}\text { Spécificité } \\
\text { physique }\end{array}$ & $\begin{array}{l}\text { - Complexité et diversité des composantes et des fonctions } \\
\text { des écosystèmes }\end{array}$ \\
\hline & $\begin{array}{l}\text { Spécificité de } \\
\text { site }\end{array}$ & $\begin{array}{l}\text { - Forte dépendance de la dynamique des écosystèmes aux } \\
\text { interactions avec leur environnement bio-géo-climatique }\end{array}$ \\
\hline & $\begin{array}{l}\text { Spécificité } \\
\text { dédiée }\end{array}$ & $\begin{array}{l}\text { - Adaptation du capital naturel déployé pour répondre à une } \\
\text { demande « sur-mesure » du développeur }\end{array}$ \\
\hline \multirow{2}{*}{$\frac{\text { Spécificité }}{\text { du capital }}$} & $\begin{array}{l}\text { Spécificité de } \\
\text { savoir faire }\end{array}$ & $\begin{array}{l}\text { - Diversité des connaissances nécessaires pour créer ou } \\
\text { restaurer les fonctions écologiques des zones humides et } \\
\text { obtenir des gains environnementaux }\end{array}$ \\
\hline & $\begin{array}{l}\text { Spécificité de } \\
\text { marque }\end{array}$ & $\begin{array}{l}\text { - Réputation des acteurs concernant leur capacité à jouer } \\
\text { leur rôle dans la transaction }\end{array}$ \\
\hline
\end{tabular}


Tableau 1 - Les différents types de spécificité des actifs engagés dans des transactions de compensation écologique

\section{La spécificité du capital naturel}

Dans le cadre de la compensation, c'est la réglementation qui définit le degré de spécificité du capital naturel en fixant les critères qui définissent l'équivalence écologique entre les pertes associées à l'impact et les gains associés à la compensation. Le régulateur utilise trois critères pour calculer l'équivalence.

Les caractéristiques physiques de l'écosystème: le terme d'écosystème aquatique renvoie à une grande variété d'habitats naturels (rivière, marais, tourbière...) qui ont des propriétés structurelles et fonctionnelles spécifiques. Dans le cadre de la compensation, l'équivalence écologique entre les gains et les pertes nécessite une définition détaillée des composantes cibles de la biodiversité et des écosystèmes (Quétier et Lavorel [2011]).

La localisation de l'action de compensation: elle influence fortement le fonctionnement des écosystèmes aquatiques (Moreno-Mateos et al. [2012]). D'une part parce qu'un écosystème ne remplit par les mêmes fonctions écosystémiques selon le lieu où il est localisé (Mitsch et Gosselink [2000]) et d'autre part parce que cette localisation influence fortement les interactions écologiques et la résilience de l'écosystème (Hanski [1998]) ${ }^{5}$.

Le fait que l'investissement doit être dédié à une demande spécifique: I'investissement dans l'écosystème aquatique que représente la compensation doit être adaptée à un impact spécifique, associé à un projet de développement particulier, et il ne peut être utilisé pour un autre impact généré par un autre projet de développement. II s'agit d'un investissement "sur-mesure ».

Les critères d'équivalence sont centraux dans l'étude des coûts de transaction. Le régulateur peut "adapter " les niveaux d'exigence autour des critères mentionnés ci-dessus pour déterminer si l'écosystème détruit et l'écosystème compensé sont équivalents (Levrel et al., 2012a ; McKenney et Kiesecker [2009] ; Quétier et Lavorel [2011]). Des niveaux de critères retenus dépendront les niveaux de coûts de transaction pour le régulateur et le professionnel de l'ingénierie écologique. En effet, plus ces critères sont stricts, plus le contrôle du respect des critères est lourd à assumer pour le régulateur. Simultanément, plus les critères sont exigeants, plus la spécificité du capital naturel déployé par le professionnel de l'ingénierie écologique pour une action de compensation est grande et plus il est difficile de redéployer cet investissement pour un autre usage que celui pour lequel il a été initialement prévu sans faire fortement augmenter les coûts. Enfin, si ces critères d'équivalence sont très contraignants, cela va prendre plus de temps au développeur de

\footnotetext{
${ }^{5}$ La question de la spécificité de site du capital naturel est par ailleurs liée à la distribution spatiale des services écosystémiques fournis pour les populations humaines qui vont pouvoir en bénéficier, même si cela n'est pas réellement pris en compte par la réglementation américaine (Flores et Thacher [2002] ; Zafonte et Hampton [2007]).
} 
pouvoir démontrer l'équivalence entre les gains et les pertes environnementales et conduire à augmenter fortement les coûts de transaction pour ce dernier.

Pour le producteur et l'acheteur, cela implique qu'ils se retrouvent dans une situation de lock-in. Aucun des deux n'a intérêt à ce que la relation qu'ils entretiennent soit rompue - au sein de cette transaction où le régulateur joue un rôle majeur - mais chacun peut chercher à exploiter la dépendance de l'autre et à s'accaparer la quasi-rente générée par de tels investissements (Klein et al. [1978]). Ainsi pour se protéger des risques de comportements opportunistes, les parties de la transaction adoptent des dispositifs de coordination contractuels relativement lourd, qui sont éloignés de ceux proposés par le marché.

\section{La spécificité du capital humain}

La compensation des impacts implique aussi des investissements dans du capital humain dont la spécificité repose sur deux éléments.

Premièrement, les actions pratiquées pour déployer l'investissement nécessitent un savoirfaire spécifique. Pour le professionnel de l'ingénierie écologique, ce savoir-faire est lié à la conception de l'écosystème et à la mise en œuvre des travaux et du suivi. Pour le développeur, ce savoir-faire concerne les capacités dont il dispose à pouvoir contrôler l'action de l'entreprise d'ingénierie. Pour le régulateur enfin, ce savoir-faire est lié à la définition de l'équivalence et aux capacités de contrôle de son respect.

De nouveau, la spécificité et donc le niveau de coûts de transaction sont largement liés aux critères d'équivalence retenus. Si ces derniers sont exigeants, la compréhension du fonctionnement de l'écosystème nécessitera un degré de savoir-faire très élevé qui ne pourra pas être intégralement redéployé vers une autre forme d'investissement. Le savoirfaire sera extrêmement spécialisé sur les mesures compensatoires pour les écosystèmes aquatiques et uniquement sur cela.

Au-delà du critère d'équivalence, un facteur qui joue fortement sur les niveaux de savoirfaire requis est le type d'action qu'il est possible de mener pour réaliser une mesure compensatoire. II existe quatre types d'action possible ${ }^{6}$ qui ne génèrent pas les mêmes gains environnementaux et ne requièrent par les mêmes niveaux de savoir-faire (Tableau 2).

\begin{tabular}{|c|c|c|c|}
\hline Action & $\begin{array}{l}\text { Gain de } \\
\text { surface }\end{array}$ & $\begin{array}{l}\text { Gain de } \\
\text { fonction }\end{array}$ & $\begin{array}{l}\text { Niveau de } \\
\text { savoir faire }\end{array}$ \\
\hline Préservation & Non & Non & - \\
\hline Amélioration & Non & Oui & + \\
\hline Création & Oui & Oui & +++ \\
\hline (réhabilitation) & Oui & Non & ++ \\
\hline Restauration (ré-établissement) & Oui & Oui & ++ \\
\hline
\end{tabular}

\footnotetext{
${ }^{6}$ Les actions de restauration concernent un écosystème qui a été dégradé et qu'il s'agit de remettre en état. Les actions de création visent à créer un nouvel écosystème, par exemple en transformant une prairie en une zone humide. Les actions d'amélioration vise à améliorer les conditions environnementales d'un écosystème en améliorant le traitement des eaux usés par exemple. Les actions de préservation visent à protéger de manière définitive un écosystème sain.
} 
Tableau 2 - Correspondance entre une action et son résultat en termes de gain net de surface et de fonctions

Deuxièmement, les acteurs impliqués dans la compensation mettent en jeu leur réputation. Cette réputation doit être considérée à l'échelle de la transaction et à une échelle plus large. A l'échelle de la transaction, cette réputation est liée à la capacité de l'acteur à jouer son rôle. Pour le professionnel de l'ingénierie écologique, sa réputation tient à sa capacité à réaliser les actions qui pourront permettre d'atteindre l'équivalence écologique en un minimum de temps, de manière à ce que le projet de développement puisse obtenir son permis. L'enjeu pour lui est donc qu'il puisse bénéficier d'une bonne réputation auprès des développeurs, pour obtenir des contrats, mais aussi auprès des régulateurs car c'est auprès de ces derniers qu'il faudra justifier de l'équivalence. Pour le régulateur il s'agit de sa crédibilité à contrôler l'application des mesures compensatoires. Ce qui est essentiel puisque le manque de crédibilité autour de la capacité de l'acteur public à mettre en œuvre un système de régulation constitue généralement un obstacle à l'investissement (Spiller et Tommasi [2005]). Cette réputation engage aussi le régulateur auprès d'un public plus large car il s'agit de sa crédibilité auprès du grand public sur sa capacité à faire respecter une politique environnementale. Une nouvelle fois, la réglementation et le critère d'équivalence jouent un rôle important pour la spécificité de marque des investissements, dans le sens où un critère très exigent augmentera les coûts de transaction.

\section{L'incertitude et la fréquence des transactions}

Les autres sources de coûts de transaction qui sont reconnues par la théorie néoinstitutionnelle sont l'incertitude (environnementale et comportementale) et la fréquence des transactions.

L'incertitude environnementale porte sur les perturbations exogènes aux décisions des contractants. Au niveau écologique, l'incertitude est une donnée essentielle pour toutes les actions mises en œuvre dans le cadre de la gestion des écosystèmes aquatiques. Malgré les progrès effectués dans la compréhension des dynamiques et de composition des communautés, la restauration d'un écosystème permet rarement de reproduire à l'identique la structure, la composition ou les fonctions d'un autre écosystème (Zedler et Callaway [1999] ; Hilderbrand et al. [2005] ; Morris et al. [2006] ; Gibbons and Lindenmayer [2007]). Les écosystèmes peuvent réagir différemment à la restauration et atteindre un état final différent d'un autre à partir des mêmes conditions initiales (Folke et al. [2004]). II n'est donc pas certain qu'une action entreprise ait les effets escomptés sur la biodiversité et les mesures compensatoires nécessitent de mettre en place un système de gestion souple et adaptatif ainsi qu'un protocole de suivi écologique permettant de s'adapter en temps réels pour atteindre les objectifs initialement fixés. Là-encore, tout cela représente une source de coûts de transaction importants pour toutes les parties.

Mais l'incertitude environnementale peut aussi se rencontrer au niveau institutionnel et réglementaire. Il existe ainsi des risques de changement des règles du jeu qui peuvent par exemple concerner l'évolution des critères d'équivalence, du fait de nouveaux cadres 
législatifs ou de décisions de justices, et avoir de fortes conséquences sur les coûts de transaction associés.

L'incertitude comportementale provient de l'opportunisme des contractants et de l'impossibilité de prévoir les comportements stratégiques qui en résultent. Dans le cadre de la compensation, le risque d'opportunisme des parties est fort tant ils n'ont aucun intérêt à connaître avec précision la qualité environnementale du bien qu'ils vont échanger, à partir du moment où le permis est délivré (Gustafsson [1998] ; Salzman et Ruhl [2000] ; Kroeger et Casey [2007]). Cela encourage les aménageurs qui cherchent à obtenir des permis à sousestimer l'impact environnemental et les investisseur dans le capital naturel à surestimer la valeur des gains produits par leurs actions de compensation (Walker et al. [2009]).

Le rôle de la fréquence des interactions entre les différentes parties, sur les coûts de transaction, est assez difficile à mesurer. Une augmentation de la fréquence des transactions accroît la probabilité, toute chose égale par ailleurs, qu'une des parties adopte un comportement opportuniste du fait d'une meilleure connaissance des sources d'incertitudes qui peuvent être utilisées de manière stratégique. Dans le même temps, la répétition d'une transaction encourage le développement de routines et d'un savoir commun entre les partenaires, et peut donc avoir une influence bénéfique sur les coûts de transaction. Les actions de manipulation des écosystèmes étant généralement soumis à des dynamiques de "learning-by-doing", nous pouvons sans trop de risque faire l'hypothèse qu'une augmentation des interactions entre les parties diminue les coûts de transaction.

D. Conséquences du niveau de coûts de transaction sur le mode d'organisation de la compensation et sur l'efficacité du système

Le mode d'organisation "historique " des mesures compensatoires est le système du permittee-responsible. Dans ce système la responsabilité de la compensation repose sur le développeur. C'est à lui de prouver au régulateur que l'investissement dans le capital naturel qui a été déployé au titre de la compensation permet bien de produire un gain environnemental équivalent aux pertes liées à son projet de développement. Le niveau d'exigence des critères d'équivalence est donc l'élément qui va fortement peser sur le niveau des coûts de transaction.

Le développeur ne possède généralement pas les compétences de génie écologique suffisantes pour mettre en place lui même l'action de compensation. II va donc faire appel à un bureau d'étude spécialisé pour mettre en place la compensation. Le besoin d'équivalence entre l'écosystème détruit et les mesures compensatoires réalisées nécessitent une planification conjointe entre le développeur et le professionnel du génie écologique. Cette planification conjointe sera d'autant plus importante que le niveau de spécificité du capital naturel sera fort et nécessitera l'adoption d'un mode organisationnel de forme hybride (Ménard [2004]). 
En, effet, alors que le marché serait incapable de mobiliser les ressources et les compétences requises pour réaliser une mesure compensatoire de manière adéquate, et que la firme entrainerait une réduction de la flexibilité, la forme hybride va permettre d'organiser les activités à travers la coordination et la coopération. Les décisions d'investissement sont donc généralement prises de manière conjointe, notamment autour du partage de la rente.

La forme hybride est par ailleurs caractérisée par l'expression forte de la concurrence. Dans le cadre de la mise en œuvre de mesures compensatoires, elle s'exprime de deux manières: entre les partenaires, qui peuvent avoir des stratégies distinctes (Coase [2000]) et avec d'autres projets qui peuvent aussi demander un permis sur un même territoire. Les partenaires sont tous les deux positionnés sur des marchés fortement concurrentiels, le marché de la construction pour les développeurs et le marché de l'ingénierie écologique pour les producteurs. Pour les partenaires le groupement de ressources et la planification conjointe sont perçus comme des moyens de mieux maîtriser l'incertitude et donc de survivre dans cet environnement fortement concurrentiel.

\section{Vers l'émergence du mitigation banking}

\section{A. Le constat de l'inefficacité du permittee-responsible pour diminuer la destruction des écosystèmes aquatiques}

A partir de la fin des années 80 , une série de rapports de terrain mirent en évidence que de nombreux sites de compensation n'avaient en fait jamais été construits et que ceux qui avaient été construits se trouvaient dans un état de dégradation important selon l'avis des écologues (National Research Council [2001] ; Government Accountability Office [2005]).

Par exemple, Kentula et collaborateurs [1992] ont étudié des permis accordés dans les Etats d'Oregon et de Washington. Pour 58 permis accordés entre 1977 et 1987 dans l'Etat d'Oregon, 82 écosystèmes aquatiques ont été détruits (74 ha) alors que 80 ont été créés (42 ha) ce qui a représenté une perte de $43 \%$ en surface d'habitats de zones humides. De même, pour 35 permis accordés entre 1980 et 1986 dans l'Etat de Washington, un déficit de 26\% d'habitats a été observé (61 ha détruits pour 45 ha créés). De plus, les écosystèmes aquatiques créés n'étaient pas les mêmes que ceux endommagés dans la plupart des cas.

Cette inefficacité de la régulation est à rapprocher du niveau très élevé des coûts de transaction qui entoure la réalisation de chaque mesure compensatoire. Le système du permittee-responsible implique que le régulateur doivent contrôler autant de mesures compensatoires qu'il existe de projets (Figure 2), ce qui conduit à augmenter fortement les niveaux de coûts de transaction et à rendre le contrôle des transactions relatives aux mesures compensatoires extrêmement difficile.

Au même moment, on assistait à une pression importante de la part de l'industrie du bâtiment qui militait pour une simplification de la procédure d'obtention des permis. Ce contexte conduisit l'administration du Président Bush (1989-1993) à proposer une série d'actions regroupées dans l'agenda "No Net Loss of Wetlands". Cet agenda a notamment rejeté l'échec de la séquence d'atténuation sur l'organisation administrative du CWA à 
l'échelle fédérale. L'origine institutionnelle de cet échec est liée à l'absence d'accord entre I'EPA et I'USACE jusqu'au début des années 90 sur: la manière dont la notion de compensation devait être entendue ; les principes qui devaient en guider la mise en œuvre (localisation par rapport au site endommagé, équivalences écologiques) ; les procédures à suivre pour réaliser ces mesures de compensation (Hough et Robertson [2008]).

C'est à partir de cette période que l'administration fédérale va appuyer l'adoption d'incitations marchandes pour la régulation du système de compensation pour les écosystèmes aquatiques (Robertson [2004]). C'est d'ailleurs en 1991 qu'apparaissent les premières expériences de mitigation banking, simultanément dans les Etats de Géorgie, d'Illinois et de Floride.

Le système du mitigation banking est la seconde forme organisationnelle permettant de réguler les mesures compensatoires (Figure 2). La plus grande innovation de ce système tient au fait que la responsabilité de la compensation repose sur un nouvel acteur qui est la banque de compensation. La banque de compensation contient généralement un ou des propriétaires fonciers, des investisseurs, une entreprise d'ingénierie écologique, à quoi peut s'ajouter éventuellement un avocat et un représentant de commerce. Le régulateur contrôle l'investissement déployé par la banque dans le capital naturel (l'écosystème) aquatique et lui attribue des "crédits" au regard des gains environnementaux obtenus à travers cet investissement. Ces crédits de compensation pourront ensuite être achetés par les développeurs ayant des projets endommageant les zones humides, sur un marché géographiquement circonscrit. A partir des années 90, deux modes de gouvernance vont cohabiter pour faire respecter les obligations de compensation associées aux impacts sur les écosystèmes aquatiques. Au cours des années 2000, suite aux critiques du GAO et de la NRC, le système des banques de compensation devient de plus important.
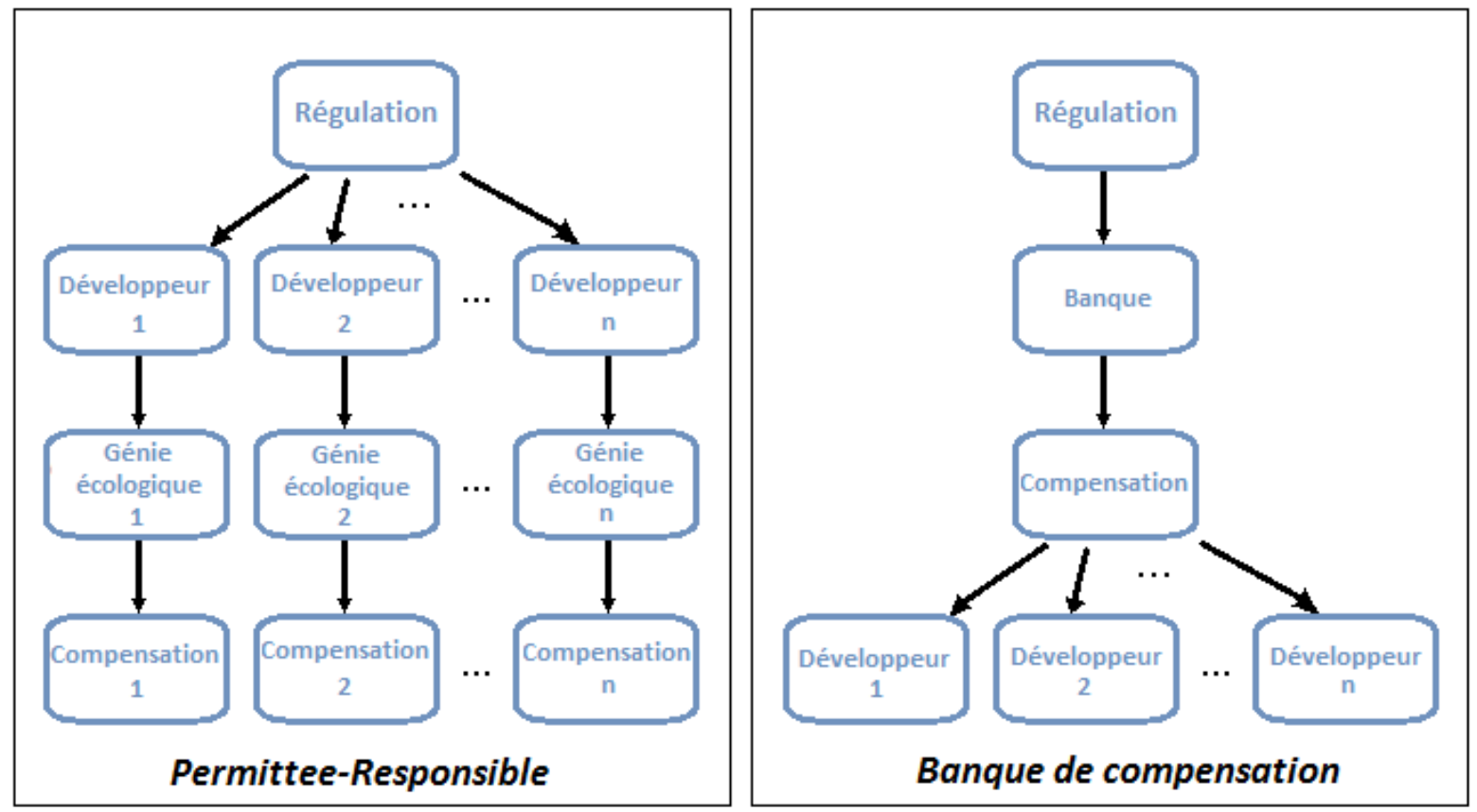

Figure 2 - Représentation de l'organisation des acteurs protagonistes dans les deux systèmes de compensation étudiés. 
B. Les innovations institutionnelles pour l'amélioration de l'efficacité de la compensation

Le transfert de la responsabilité du développeur à la banque de compensation s'accompagne d'un changement des droits de propriété ${ }^{7}$.

Par ailleurs, pour que les banques de compensation se développent, les administrations en charge du système de compensation ont cherché à réduire l'incertitude environnementale en stabilisant l'environnement institutionnel grâce à l'adoption d'un certain nombre d'actions qui se sont étalées entre le début des années 90 et 2008 (Levrel [2012] ; Scemama et Levrel [2012]):

- la signature d'un Memorandum Of Agreement (MOA) en 1990 entre I'USACE et l'EPA en vue de stabiliser les règles des procédures d'évitement, de réduction et de compensation ${ }^{8}$;

- la mise en place, à partir de 1995, d'un système d'attribution de crédits aux banques de compensation sur la base de considérations écologiques objectives et quantifiables, en précisant les standards à partir desquels les compensations pouvaient être évaluées ;

- la clarification des préférences administratives: pour le système des banques de compensation plutôt que pour le système de permis ; pour les actions de restauration plutôt que pour les actions d'amélioration, de création et de préservation ; pour les compensations "in kind" correspondant au respect d'une équivalence "écologique" ; pour les compensations à proximité des zones d'impacts pour le système de permis et dans tous les cas pour le respect d'une logique écosystémique par bassin versant ;

- la création d'aires de services dans lesquelles les banques doivent réaliser les transactions et qui correspondent donc aux marchés dans lesquels un crédit peut être vendu ;

- la mise en place de ratios d'équivalence permettant de pondérer les efforts de compensation en fonction de leurs caractéristiques (distance par rapport à la zone d'impact ou type de compensation choisi).

Tous ces éléments étaient traités de manière non homogène jusque dans les années 90 et étaient à l'origine d'une incertitude importante sur les procédures à suivre et les objectifs à atteindre dans le cadre des mesures compensatoires. Les enseignements de la théorie néoinstitutionnelle permettent d'étudier les impacts de ces changements institutionnels sur l'efficacité des modes d'organisations et sur les caractéristiques des transactions (Annexe 1.B).

C. L'évolution des coûts de transaction liée à de nouvelles contraintes sur la spécificité des actifs

\footnotetext{
${ }^{7}$ Les banques de compensation placent le terrain qu'ils restaurent sous un statut de servitude environnementale perpétuelle, c'est à dire qu'elle transfert une partie de ses droits de propriété sur le terrain à un trustee, une organisation chargée de la protection de l'environnement ou le gouvernement (comté, état ou fédéral) afin qu'il garantisse sa conservation.

${ }^{8}$ En 1990, l'USACE organise aussi un workshop sur la question des banques de compensation et entre 1990 et 1995 commande un grand nombre d'expertises, ce qui va créer un climat de confiance propice à l'émergence de ces banques (Hough et Robertson [2008]).
} 
La stimulation de l'investissement dans le système des banques de compensation correspond aussi à un phénomène d'adaptation des acteurs au changement de l'environnement institutionnel, qui s'est traduit par une modification du degré de spécificité des actifs de manière à aligner les attributs des transactions et le mode de gouvernance. Les adaptations organisationnelles des acteurs ont conduit à une baisse générale de la spécificité des actifs (Tableau 4).

\begin{tabular}{|l|l|c|l|}
\hline \multicolumn{1}{|c|}{ Permittee-responsible } & $\begin{array}{l}\Delta \text { Coûts de } \\
\text { transaction }\end{array}$ & \multicolumn{1}{|c|}{ Mitigation banking } \\
\hline $\begin{array}{l}\text { Spécificité de } \\
\text { site du capital } \\
\text { naturel }\end{array}$ & $\begin{array}{l}\text { Compensation à réaliser sur le } \\
\text { site de l'impact }\end{array}$ & $>$ & $\begin{array}{l}\text { Compensation dans une } \\
\text { zone de service }\end{array}$ \\
\hline $\begin{array}{l}\text { Spécificité } \\
\text { physique du } \\
\text { capital naturel }\end{array}$ & $\begin{array}{l}\text { Equivalence discutée au cas } \\
\text { par cas }\end{array}$ & $\mathbf{P}$ & $\begin{array}{l}\text { Equivalence estimée en } \\
\text { termes de crédits }\end{array}$ \\
\hline $\begin{array}{l}\text { Capital naturel } \\
\text { dédié }\end{array}$ & Un projet de développement & $>$ & $\begin{array}{l}\text { Plusieurs projets de } \\
\text { développement }\end{array}$ \\
\hline $\begin{array}{l}\text { Spécificité du } \\
\text { savoir faire }\end{array}$ & $\begin{array}{l}\text { Connaissances spécifiques à } \\
\text { une mesure compensatoire }\end{array}$ & $>$ & $\begin{array}{l}\text { Connaissances spécifiques à } \\
\text { toute la banque }\end{array}$ \\
\hline $\begin{array}{l}\text { Spécificité de } \\
\text { marque }\end{array}$ & $\begin{array}{l}\text { Réputation mise en jeu à } \\
\text { chaque impact }\end{array}$ & $>$ & $\begin{array}{l}\text { Réputation mise en jeu pour } \\
\text { une banque }\end{array}$ \\
\hline
\end{tabular}

Tableau 3 - Conséquences de l'évolution des différentes formes de spécificité des actifs entre les deux systèmes de compensation sur les coûts de transaction

La principale adaptation est liée au fait qu'un producteur puisse mettre en place une action de compensation pour compenser les impacts de plusieurs développeurs, diminuant le degré de spécificité des actifs suivant plusieurs dimensions.

Premièrement, une diminution du caractère dédié du capital naturel: le système des banques de compensation correspond à une augmentation de la taille des marchés par rapport au permittee-responsible et chaque banque de compensation peut avoir plusieurs clients différents à partir d'une même action de compensation.

Deuxièmement, une diminution de la spécificité de site: la création d'un système de crédit d'écosystème aquatique implique qu'on s'affranchisse en partie du contexte géophysique. En effet, le critère de proximité de la mesure compensatoire n'a plus de sens dans le système du marché de crédit. Une banque pouvant compenser plusieurs impacts, ces derniers ne seront pas situés au même endroit et évidemment pas sur le site de la banque. La dimension spatiale est donc essentielle dans le problème d'interchangeabilité des unités de biodiversité. Des solutions sont proposées pour intégrer la spécificité de site dans le calcul des crédits alloués à une banque à l'aide de méthodes basées sur les principes de génétique des populations (Bruggeman et al. [2005] ; Scribner et al. [2005]). Cependant de telles études restent des propositions qui ne trouvent pas d'échos dans la pratique, tant les coûts de transaction qu'elles entraîneraient seraient importants. Le système américain des banques de compensation d'écosystèmes aquatiques a simplement choisi de restreindre les échanges à certaines zones géographiques: les aires de service qui limitent la zone dans laquelle une banque peut vendre des crédits de compensation. 
Troisièmement, une diminution de la spécificité du capital humain. D'une part, du fait de la transférabilité des savoir-faire d'une transaction de compensation vers une autre, à partir du moment où elles concernent la même banque. D'autre part, la spécificité de marque associée à une banque de compensation n'est pas remise en jeu à chaque compensation, mais seulement au moment de l'autorisation de la création de la banque et au moment de l'attribution des crédits. Enfin, parce que le transfert de responsabilité de la compensation à la banque élimine, pour le développeur, les besoins de maîtriser un savoir-faire qui ne fait pas partie de son corps de métier et que ce dernier ne sélectionne pas une banque de compensation au regard de sa réputation mais des crédits dont elle dispose dans une aire de service donnée.

Pour ce qui concerne la spécificité physique, les choses sont plus nuancées. L'innovation institutionnelle majeure à réaliser pour pouvoir faire émerger un marché de la compensation a été de créer des unités de crédit de compensation standards pour des impacts sur des écosystèmes aquatiques. Ceci correspond à une standardisation du critère d'équivalence, au moins à l'échelle d'une aire de service. Mais cette standardisation, si elle peut être synonyme de réduction de la spécificité de l'actif compensé, est aussi une source de plus grande rigueur dans les actions menées. Ainsi, l'attribution des crédits et les critères écologiques sur lesquels ils reposent, sont mieux définis dans le cas des banques de compensation que pour les systèmes de permis. A titre d'exemple, dans le district de Chicago, les crédits sont distribués en quatre phases : (1) $30 \%$ des crédits sont délivrés en même temps que le permis d'autorisation de mise en place de la banque, (2) $20 \%$ sont délivrés une fois que le banquier apporte la preuve que la surface des eaux souterraines a été mesurée à moins de $30 \mathrm{~cm}$ de la surface du sol pendant plus de deux semaines entre Mai et Octobre, (3) $20 \%$ sont délivrés une fois que le banquier apporte la preuve qu'un ensemble "approprié" d'espèces végétales natives ont bien été plantées et (4) les $30 \%$ restant sont délivrés quand le banquier fournit la preuve que le site répond bien à une liste de critères écologiques plus précis (Robertson et Hayden [2008]). Ces critères d'obtention des crédits ont par ailleurs tendances à se renforcer depuis 2008 et inclus aujourd'hui des indicateurs de micro-organismes des sols, de présences/absences d'espèces inféodées aux zones humides et de taux de couverture du sol.

On peut penser que pour réellement mesurer l'équivalence écologique entre un site endommagé et sa mesure compensatoire, il faudrait déployer des actifs humains bien plus spécifiques que ceux déployés aujourd'hui. Pour autant, d'un point de vue relatif, on observe un niveau d'exigence bien supérieur dans le système des banques de compensation que dans le système de permittee responsible, pour une simple question de faisabilité du contrôle associé.

\section{L'évolution de l'incertitude et de la fréquence des transactions}

Les innovations institutionnelles ont eu pour conséquences de changer l'efficacité relative des modes d'organisation. 
L'apparition des banques a eu un effet d'entraînement pour ce qui concerne la réduction des incertitudes institutionnelles. En effet, la création du marché et son expansion ont conduit les nouvelles banques à demander une clarification toujours plus forte des "règles du jeu" et obligé les administrations régulatrices à s'organiser de manière plus efficace, en établissant en particulier un référentiel précis autour des crédits de compensation. En effet, les aléas autour de l'évaluation de l'équivalence sont certainement la plus grande source d'incertitude pour les banques de compensation (Moilanen et al. [2009]). C'est la réglementation qui conditionne le niveau de demande et de prix pour les crédits de compensation à travers l'obligation imposée à un certain nombre d'activités de mettre en place des mesures compensatoires, mais aussi le niveau et la qualité de l'offre - exigence concernant les critères d'équivalence écologique pour les crédits échangés - par le contrôle et la validation de ces mesures (Robertson [2008]). La stabilisation des règles de régulation a permis une diminution de l'incertitude institutionnelle, une diminution des coûts de transaction, et finalement conduit à stimuler l'investissement. En effet, les risques associés à l'investissement dans le capital naturel sont apparus comme plus raisonnables aux yeux des acteurs souhaitant développer des banques de compensation.

Dans le même temps, le système des banques de compensation a permis de réduire fortement les coûts de transaction supportés par l'administration. En effet, pour les acteurs régulateurs, le mécanisme permittee-responsible représente une charge importante liée au nombre de dossiers à étudier. Cela génère des risques de comportements opportunistes de la part des aménageurs qui, sachant que les dossiers sont peu ou mal contrôlés par les autorités, vont avoir tendance à minimiser le coût des actions de compensation. En transférant la responsabilité du no net loss aux banques, les autorités de régulation s'assurent de concentrer les actions de compensation et réduisent ainsi fortement les ressources nécessaires pour le contrôle (Marsh and Acker [1992] ; Etchart [1995] ; Figure 2). Par ailleurs, la responsabilité des actions de compensation ayant été concentrée dans les mains d'un petit nombre de banques, la fréquence des transactions entre les banques et les administrations a fortement augmentée, ce qui favorise le développement de routines, d'habitudes contractuelles, et permet l'émergence d'un climat de confiance qui réduit le besoin de mécanismes de coordination formels, notamment concernant le contrôle de l'efficacité de certains protocoles de restauration éprouvés sur le terrain.

En plus d'une diminution de l'incertitude institutionnelle et comportementale, le système des banques de compensation permet une diminution notoire de l'incertitude associée aux variabilités naturelles. Tout d'abord car les banques de compensation permettent d'éviter les pertes temporaires ou permanentes de services écosystèmiques du fait que les crédits sont attribués pour la vente au fur et à mesure que les objectifs environnementaux sont remplis ${ }^{9}$ (Walker et al. [2009] ; Bekessy et al. [2010]). Ensuite parce que la concentration des

\footnotetext{
9 Dans le cas des mesures compensatoires, il n'est cependant pas possible d'attendre que toutes les potentialités écologiques de l'écosystème restauré s'expriment pour donner au développeur l'autorisation de lancer son projet de développement, sans quoi il devrait attendre des années avant d'avoir le moindre retour
} 
actions de compensation permet d'avoir des projets de restauration écologique de plus grande envergure, qui affichent de meilleures probabilité de succès que les projets mis en place sur de petites échelles spatiales (Brown and Veneman [2001]). L'étude de l'efficacité écologique des actions de restauration pour les écosystèmes aquatiques montre par exemple l'existence d'une forte corrélation entre la taille des surfaces restaurées et l'efficacité écologique, avec un taux de succès de $100 \%$ pour les surfaces de plus de 100 hectares (Moreno-Mateos et al. [2012]).

La baisse des coûts de transaction pour l'administration, combiné à l'augmentation supposée de l'efficacité écologique associé à ce système de banque de compensation, a entraîné une forte évolution vers ce mode d'organisation (Tableau 3).

\begin{tabular}{|l|l|c|l|}
\hline & \multicolumn{1}{|c|}{ Permittee-responsible } & $\begin{array}{l}\Delta \text { Coûts de } \\
\text { transaction }\end{array}$ & \multicolumn{1}{|c|}{ Mitigation banking } \\
\hline $\begin{array}{l}\text { Incertitude } \\
\text { réglementaire }\end{array}$ & $\begin{array}{l}\text { Critère d'équivalence au cas } \\
\text { par cas }\end{array}$ & $>$ & $\begin{array}{l}\text { Critère d'équivalence stable } \\
\text { et précis }\end{array}$ \\
\hline $\begin{array}{l}\text { Incertitude liée à } \\
\text { l'environnement }\end{array}$ & $\begin{array}{l}\text { Actions sur une surface liée à } \\
\text { celle de l'impact } \\
\text { Compensation débutée au } \\
\text { moment de l'impact }\end{array}$ & $>$ & $\begin{array}{l}\text { Actions sur de plus grandes } \\
\text { surface } \\
\text { Compensation débutée } \\
\text { avant l'impact }\end{array}$ \\
\hline $\begin{array}{l}\text { Incertitude } \\
\text { comportementale }\end{array}$ & $\begin{array}{l}\text { Mesures compensatoires peu } \\
\text { ou pas contrôlées }\end{array}$ & $>$ & $\begin{array}{l}\text { Contrôle plus important des } \\
\text { banques }\end{array}$ \\
\hline $\begin{array}{l}\text { Fréquence des } \\
\text { transactions }\end{array}$ & $\begin{array}{l}\text { Autant de partenaires qu'il y } \\
\text { a de transaction }\end{array}$ & $>$ & $\begin{array}{l}\text { Réduction du nombre de } \\
\text { partenaires }\end{array}$ \\
\hline
\end{tabular}

Tableau 4 - Conséquences de l'évolution de l'incertitude et de la fréquence des transactions entre les deux systèmes de compensation sur les coûts de transaction

\section{Discussion}

Les premières réponses institutionnelles au problème de l'érosion du capital naturel ont été construites sur les bases des travaux de Hardin [1968], qui dans son article intitulé Tragedy of the commons, déclarait que l'absence de droits de propriété bien définis sur les ressources naturelles conduisait ces dernières à être surexploitées. Depuis cette époque, les théories et les pratiques entourant les services écosystémiques ont en partie évolué dans le sens de la création ou la clarification de droits de propriété - notamment privés - en vue de permettre la marchandisation de ces derniers et de réguler leur usage. On a ainsi pu observer l'apparition des marchés pour encadrer les émissions de gaz à effet de serre (Barker et al. [2001] ; Bayon [2004]), les émissions de dioxyde de souffre (Stavins [1998]), les prélèvements d'eau (Libecap [2011] ; Garrick et al. [2013]) ou l'usage des stocks de pêche

sur investissement. C'est pourquoi des crédits sont attribués dès que le terrain est sécurisé, qu'une servitude environnementale est adoptée et que des fonds assurantiels ont été mobilisés. De ce fait, il existe ce que l'on nomme des pertes temporaires qui correspondent au décalage entre les pertes de services écosystémiques observées sur le site où l'impact a eu lieu et les gains "en devenir" sur le site où les mesures compensatoires ont été réalisées. C'est pourquoi il faut nuancer ce principe selon lequel les compensations sont réalisées avant les impacts. 
(Thébaud [2012]). Dans le domaine de la biodiversité, on a assisté aux Etats-Unis à l'émergence d'un marché de la compensation des zones humides.

Le processus de marchandisation de certaines composantes de la biodiversité nécessite la création et/ou la modification de règles institutionnelles qui autorisent les transactions sur des marchés de services écosystèmiques (Bayon [2004]). Des changements institutionnels ont donc été appliqués, de façon à soumettre la transaction aux mécanismes de coordination du marché (plus d'incitations et moins de contrôle administratif), en jouant sur les caractéristiques des transactions, notamment à travers l'exigence environnementale des règles d'échange.

L'analyse néo-institutionnelle de ce processus d'émergence des banques de compensation permet de dire que, toutes choses égales par ailleurs, si l'on observe aujourd'hui un accroissement fort du mode de gouvernance "marchand" par rapport au mode de gouvernance "administré" c'est que les dispositifs de coordination du premier sont plus efficaces - en termes de coûts de transaction - quand il s'agit d'encadrer les transaction liées à la compensation pour les écosystèmes aquatiques. On constate d'ailleurs que d'autres tentatives de création de marché de la biodiversité n'ont pas connu le même succès, probablement parce que les coûts de transaction associés à ces marchés étaient trop élevés. On peut citer en exemple le "species banking" (Madsen et al. [2011]) qui n'a pas connu le même succès que le marché des écosystèmes aquatiques, sans doute parce que la spécificité de l'actif y était beaucoup plus importante (un système de crédit pour chaque espèce).

La régulation profite ainsi du comportement calculateur des parties de la transaction qui vont chercher à inscrire leur relation dans l'arrangement institutionnel qui minimise les coûts de transaction (Figure 3).

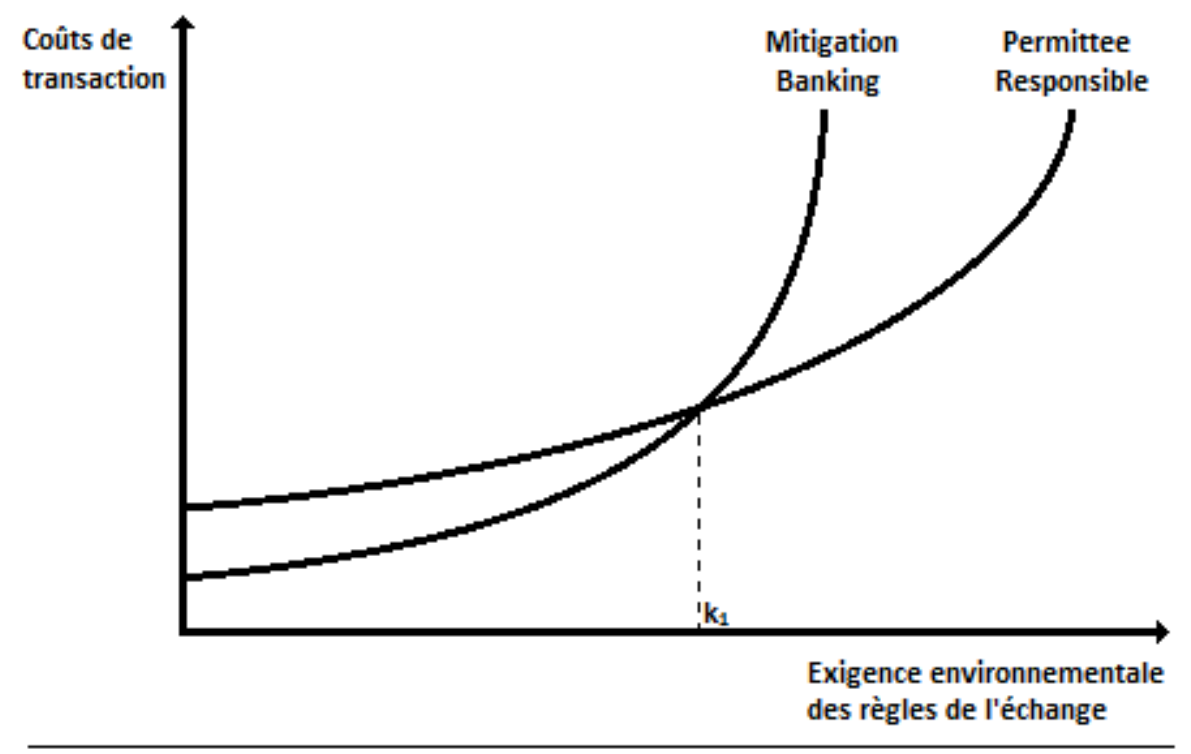

Figure 3 - Arbitrage entre les deux systèmes de compensation selon l'exigence environnementale des règles de l'échange. Lorsque le système de classification des crédits est générique et que la compensation peut se faire à une distance éloignée du lieu de l'impact $\left(k<k_{1}\right)$, les parties de la transaction choisiront plus facilement d'adopter le système du mitigation banking. 
Pour autant, la création d'un marché de compensation d'écosystème aquatique comporte de nombreux risques.

En premier, elle nécessite la constitution d'unités d'échange suffisamment homogènes pour pouvoir être interchangeables en qualité et en caractéristiques, sans quoi il ne peut y avoir de marché. Contrairement aux crédits carbone, qui sont construits sur la base d'une unité unique et quantifiable, les valeurs de la biodiversité sont complexes à mesurer, en particulier si l'on cherche à décrire précisément des services écosystèmiques à travers ces unités (Salzman et Ruhl [2002]). II existe ainsi une tension entre un objectif de conservation des écosystèmes aquatiques et un besoin de réduction de la spécificité physique du capital naturel. D'un côté les crédits qui vont être échangés devraient désigner l'ensemble des composantes ou des fonctions de la biodiversité sans quoi il existe un risque de voir disparaître celles qui ne seraient pas inclues dans l'unité d'échange (ten Kate et al. [2004]). D'un autre côté, plus le crédit désignera un écosystème aquatique générique, plus le crédit en question sera proche de l'hypothèse d'homogénéité posée par Arrow et Debreu [1954], plus les coûts de transaction seront réduit et plus il sera facile de trouver un acheteur.

Dans la même veine, la spécificité de site du capital naturel va aussi être réduite, à travers le recours aux banques de compensation, par le fait que leurs stratégies d'investissement conduisent à une redistribution spatiale des écosystèmes aquatiques. En effet, même s'il existe des limites géographiques au marché des crédits de compensation, fondées sur des critères écosystémiques, les banques ont tendance à investir dans des zones rurales où le foncier n'est pas cher, pour compenser des impacts qui ont principalement lieu dans des zones urbaines. Elles vont ainsi contribuer à une réorganisation des paysages avec une "migration" des écosystèmes aquatiques depuis les régions urbaines vers les régions rurales, phénomène décrié comme étant une cause de création de disparité et d'iniquité sociale, mais aussi comme une source de perte de spécificité de site de l'actif compensé (King et Herbert [1997] ; Ruhl et Salzman [2006]).

En théorie, plus on a recours aux mécanismes marchands, plus il est nécessaire de réduire la spécificité et plus on s'éloigne du principe de durabilité forte. Ce que suggère le tableau 5 , c'est que les modifications institutionnelles nécessaires pour voir apparaître des modes de gouvernance plus proche du marché, et ainsi permettre d'améliorer l'efficacité institutionnelle du mécanisme, ne vont pas dans le sens de l'amélioration de son efficacité environnementale. L'analyse néo-institutionnelle de ces tensions nous permet de poser un nouveau regard sur la définition de l'objectif de no net loss. La question essentielle qui se pose lors de la création d'un marché de biodiversité, est celle de l'unité qui sera utilisée pour les échanges et qui renvoie à différents niveaux de no net loss auxquels, correspondant euxmêmes à différents niveaux de durabilité (Levrel et al. [2012b] et Tableau 5).

Ainsi, une étude récente montre que "selon les standards réglementaires, $98,3 \%$ des banques autorisées à vendre des crédits ont atteint ou vont atteindre ces standards de performance", preuve d'une efficacité écologique au regard de critères réglementaires (Denisoff et Urban [2012]). Mais quels sont ces standards et comment les rapprocher 
d'objectifs de durabilité précis concernant le no net loss d'écosystèmes aquatiques? A chaque niveau de durabilité on peut associer un niveau de spécificité du capital naturel.

\begin{tabular}{|l|l|l|l|l|}
\hline $\begin{array}{l}\text { Durabilité et } \\
\text { qualité du "no net } \\
\text { loss" }\end{array}$ & Faible & Forte \\
\hline $\begin{array}{l}\text { Spécificité de site } \\
\text { du capital naturel }\end{array}$ & $\begin{array}{l}\text { Off-site dans le } \\
\text { Pays }\end{array}$ & $\begin{array}{l}\text { Off-site dans le } \\
\text { District }\end{array}$ & $\begin{array}{l}\text { Off-site dans le } \\
\text { County }\end{array}$ & On-site \\
\hline $\begin{array}{l}\text { Spécificité } \\
\text { physique du } \\
\text { capital naturel }\end{array}$ & $\begin{array}{l}\text { Pas de perte } \\
\text { nette de bien- }\end{array}$ & $\begin{array}{l}\text { Pas de perte } \\
\text { nette de capital } \\
\text { naturel }\end{array}$ & $\begin{array}{l}\text { Pas de perte } \\
\text { nette } \\
\text { services } \\
\text { écosystèmiques }\end{array}$ & In-kind \\
\hline $\begin{array}{l}\text { Spécificité du } \\
\text { capital humain }\end{array}$ & Objectif de moyen & \multicolumn{4}{|l|}{ Objectif de résultats } \\
\hline
\end{tabular}

Tableau 5 - Durabilité et spécificité de capital

Pour mettre en place un marché de la compensation qui ait les caractéristiques d'un "marché de la qualité" permettant de générer des transactions qui ne sacrifient pas la spécificité de l'actif que représente la biodiversité, l'Etat doit maintenir un haut niveau de régulation de ce marché de la compensation. Il doit notamment veiller à faire évoluer continuellement le système d'évaluation de cette qualité, de manière à permettre aux producteurs qui doivent assumer des coûts de production élevés de pouvoir faire valoir un gain de qualité auprès de la demande dans le système de concurrence en place. Il doit par ailleurs veiller au maintien d'un cadre réglementaire et de contrôle strict qui va pouvoir peser sur l'incertitude institutionnelle et garantir un certain niveau de spécificité de l'actif naturel à mobiliser. II faut par ailleurs souligner que les investissements publics dans le contrôle et la régulation de ce marché vont conduire, par un effet multiplicateur, à des investissements privés dans la biodiversité du fait de l'identification de nouveaux marchés dans un contexte institutionnel stabilisé. Or, pour envisager des investissements dans la conservation de la biodiversité qui puissent avoir du sens à de larges échelles spatiales et contrebalancer l'érosion globale que subit le vivant, il est intéressant de pouvoir s'appuyer sur un tel levier d'action.

\section{Conclusion}

Au cours de cet article, nous avons étudié le fait que les caractéristiques des transactions qui mettent en jeu des investissements dans du capital naturel sont originales, qu'elles pèsent sur les modes d'organisation et l'efficacité des changements institutionnels et que cela conditionne in fine la qualité de la compensation. Cette hypothèse a été testée à travers l'étude de l'émergence des banques de compensation pour les écosystèmes aquatiques aux USA. A travers notre analyse, nous pouvons donc tirer un certain nombre d'enseignements :

- Premièrement, nous avons vu que le capital naturel a des caractéristiques originales qui impliquent des transaction dont les attributs se révèlent être des éléments à l'origine de coûts de transaction importants pour les acteurs. 
- Deuxièmement, il apparait que ces caractéristiques impliquent des adaptations organisationnelles qui tendent vers des formes hybrides complexes dont les dispositifs de coordinations sont les plus adaptés pour encadrer de telles transactions. Ces formes hybrides pourraient représenter des formes organisationnelles originales et encore peu étudiées du point de vue de la théorie néo-institutionnelle.

Si ces conclusions ont une portée plus théorique, nous pouvons aussi tirer des enseignements pertinents pour les politiques de compensation environnementale :

- Premièrement, la volonté d'inscrire les transactions dans un cadre marchand entraîne le risque de voir les parties chercher à aligner les caractéristiques de la transaction sur cette forme organisationnelle, c'est à dire réduire la spécificité du capital naturel que représentent les écosystèmes aquatiques et donc diminuer la qualité de la compensation environnementale.

- Deuxièmement, la conséquence directe de ce risque de diminution de la spécificité du capital naturel est qu'il est nécessaire de maintenir un contrôle étatique fort dans ce type de marché à travers un contexte institutionnel contraignant (règles d'équivalence, suivis, sanctions, etc.).

Ces enseignements doivent permettre de répondre aux questions qui se posent au niveau français face à l'arrivée des banques de compensation. Il est ainsi probable que le semi échec de la CDC Biodiversité ${ }^{10}$ puisse être expliqué grâce aux éléments analysés ici. La création d'un système marchand s'est faite sans création d'un environnement institutionnel adapté, ni réflexion sur le lien entre niveau d'exigence de l'équivalence et caractéristiques de la transaction associée, ce qui pourrait finalement conduire à son effondrement. De nouvelles expérimentations ont été lancées par le ministère (MEDDE), à partir desquelles il sera possible de proposer une analyse plus fine des questions soulevées ici ${ }^{11}$.

\footnotetext{
${ }^{10}$ En 2008, la CDC Biodiversité a initié le premier projet de compensation par l'offre au cœur de la réserve naturelle des Coussouls de Crau, près de Fos-sur-Mer. Pour une analyse institutionnelle du mécanisme, voir Chabran et Napoléone [2013].

${ }^{11}$ Voir: http://www.developpement-durable.gouv.fr/Appel-a-projet-pour-tester-un.html
} 


\section{Annexes}

\section{A. Présentation de la théorie néo-institutionnelle}

Le point de départ de l'économie néo-institutionnelle est que les agents ne sont pas considérés comme étant rationnels, mais comme ayant une rationalité limitée ${ }^{12}$ et un comportement opportuniste ${ }^{13}$. La rationalité limitée des agents économiques impliqués dans une transaction rend impossible la rédaction d'un contrat qui soit complet. De plus I'hypothèse d'opportunisme met en exergue la nécessité de construire des contrats qui constituent des engagements suffisamment crédibles pour encadrer le comportement des agents et ainsi sécuriser l'objet de la transaction, c'est ce qui est à l'origine des coûts de transaction. C'est pourquoi les échanges sont complétés par d'autres mécanismes de coordination, ensemble qui constitue le mode de gouvernance ${ }^{14}$. II en existe trois types: le marché, la hiérarchie et les formes hybrides ${ }^{15}$. Le problème qui se pose aux contractants est de choisir la structure de gouvernance la plus efficace, c'est à dire celle qui minimise les coûts de transaction et les coûts de production. Toutefois la théorie retient que dans la majorité des cas, ce sont des considérations en termes de coûts de transaction qui permettent d'expliquer le choix des modes d'organisation (Saussier [1997]) ${ }^{16}$.

L'efficacité relative des modes d'organisation dépend de leur système de coordination: dans le marché, la coordination est assurée par le système de prix, alors que dans une organisation hiérarchique la coordination est assurée par un contrôle administratif. Les limites du marché pour coordonner l'activité économique et l'allocation des ressources ont été mises en évidence par Coase [1937] qui a montré l'existence de coûts de transaction liés au marché : coût de détermination du juste prix, coût de négociation et de contractualisation. Ce dernier souligne qu'il existe un niveau de coûts de transaction pour lequel il sera plus intéressant d'avoir recours à la coordination par l'organisation hiérarchique plutôt que par le système de prix.

Pour Coase, les coûts de transaction sont ainsi déterminants dans la décision d'intégrer verticalement un atelier de production au sein d'une organisation (qu'elle soit publique ou privée). Ce dernier va même plus loin en disant qu'en l'absence de coûts de transaction,

\footnotetext{
${ }^{12}$ Les agents sont incapables d'anticiper ex ante tous les états de la Nature. Cette hypothèse a été d'abord posée par Simon [1961] qui qualifiait les agents économiques comme ayant un comportement "intentionnellement rationnel, mais seulement de façon limitée" (Simon [1961], p24).

${ }^{13}$ L'hypothèse d'opportunisme des agents correspond à un comportement stratégique des agents par lequel ils vont chercher à exploiter les failles de la relation contractuelle pour leur propre intérêt (Williamson [1993]).

${ }^{14}$ Dans cet article nous utiliserons indifféremment les termes mode de gouvernance et mode d'organisation.

${ }^{15}$ Ici, le marché est le même concept que celui utilisé par l'économie néo-classique, c'est-à-dire un système de contrôle coordonné par le signal prix. La hiérarchie se réfère à la structure hiérarchique et généralement centralisée de l'entreprise intégrée (public ou privé) qui est fondée sur des liens de subordination entre les agents. Les formes hybrides couvrent une variété de dispositifs tels que les contrats à long terme, les alliances, les franchises, les marques ou des licences de fabrication, etc.

${ }^{16}$ L'incertitude sur les états futurs de la nature intervient aussi dans cet arbitrage, aussi les points de basculement d'une forme vers une autre sont flou ce qui rend possible la coexistence de modes d'organisation alternatifs au voisinage de ces points (Ménard [2012]).
} 
dans un contexte concurrentiel, l'allocation des droits de propriété se fait automatiquement de manière optimale par la négociation directe entre les agents et ce, quelle que soit la répartition initiale de ces droits (Coase [1960]). Sans coûts de transaction, la question du choix organisationnel pour encadrer une transaction ne se pose plus.

L'importance des coûts de transaction pour expliquer l'émergence des modes d'organisation alternatifs au marché, sera reprise par Williamson [1985 et 1996] qui explique le choix du mode de gouvernance d'une activité en fonction des caractéristiques des transactions. Le fait que l'analyse des modes d'organisation nécessite la comparaison directe des coûts de transaction a été l'objet de critiques importantes notamment du fait que ces coûts sont difficiles à observer et à mesurer dans une structure existante, mais aussi qu'ils ne peuvent pas être étudiés pour des formes alternatives qui ne sont pas encore existantes (Masten et al. [1991]). Pour faire face à ces critiques, la théorie s'est attachée à relier les coûts d'une transaction avec certaines de ses caractéristiques et à ensuite poser les fondements de la discussion sur ces caractéristiques observables. Ainsi il existe trois sources de coûts de transaction : l'incertitude qui entoure la transaction, la fréquence à laquelle la transaction est réalisée et le degré de spécificité des actifs impliqués dans la transaction. C'est à partir de discussions sur ces caractéristiques que la théorie compare les différents modes de gouvernance (Figure 1).

L'approche néo-institutionnelle a ainsi trois fondements majeures qui justifient qu'on utilise ce cadre pour notre analyse. La première concerne l'objet d'investigation qui porte sur la transaction définie comme un transfert de droits, mettant en exergue les aspects relationnels entre les parties. La deuxième porte sur le rôle de l'efficience qui confère aux parties de la transaction un comportement calculateur envers les coûts de transaction, ce qui les conduit à comparer les modes de gouvernance entre eux pour trouver l'arrangement le mieux adapté, qui va du marché à l'organisation hiérarchique en passant par des modes de gouvernance hybrides (Figure 4). Enfin la troisième différence porte sur l'importance donnée à l'environnement institutionnel, puisque ce dernier par le biais de règles et de dispositifs encadre les transactions (Ménard [2000]). 


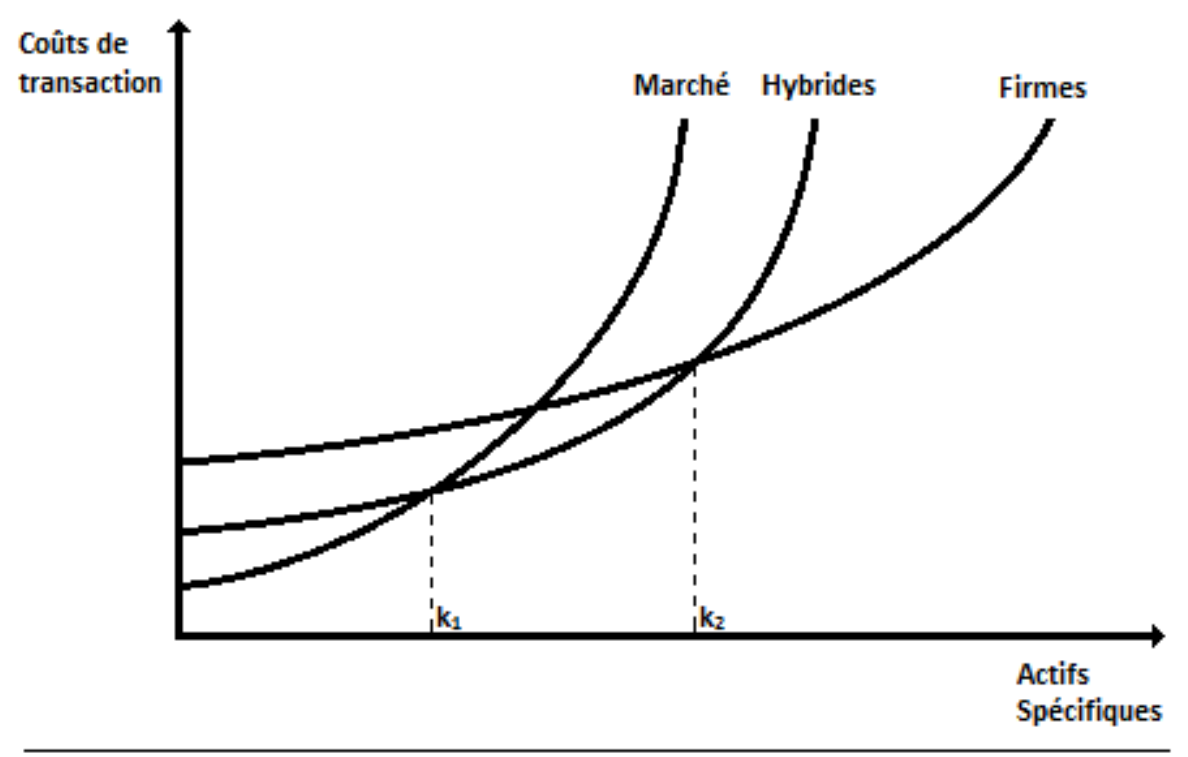

Figure 4 - L'arbitrage en présence des trois grandes familles d'organisation (Williamson [1991]). Lorsque les investissements sont génériques $\left(k<k_{1}\right)$, les acteurs s'orienteront vers le marché pour encadrer leur relation. Lorsque le degré de spécificité des investissements est élevé $\left(k>k_{2}\right)$, c'est la solution de l'intégration verticale qui sera retenue. Entre les deux $\left(\mathrm{k}_{1}<\mathrm{k}<\mathrm{k}_{2}\right)$, se situe la zone où dominent les arrangements "hybrides".

\section{B. Influence des institutions sur les modes d'organisation}

Un des volets importants de l'économie néo-institutionnelle, a été développé par Douglass North et concerne l'étude de l'environnement institutionnel et de son influence sur l'efficacité des modes d'organisation (North [1991]). Les institutions sont vues comme "les contraintes créées par l'homme pour structurer les interactions politiques, économiques et sociales" (North [1991], p 97). Elles fournissent ainsi la structure incitative d'une économie et constituent le cadre dans lequel les organisations peuvent évoluer, elles posent les limites qui définissent l'ensemble des alternatives envisageables. Ce sont sur elles que les politiques publiques vont pouvoir agir pour inciter à l'émergence d'une nouvelle forme d'organisation à même d'investir dans le capital naturel. En effet, le changement institutionnel modifie l'efficacité des modes d'organisation en pesant sur les coûts de transaction (Ménard [2003]). Dans la pratique, les interactions entre une forme d'organisation optimale et son environnement institutionnel peut être considérée de deux manières.

Premièrement, certains travaux s'intéressent à la relation qui existe entre les caractéristiques des institutions et l'efficacité des modes de gouvernance. On suppose que plus il existe de dispositifs crédibles garantissant le respect de la propriété et des engagements, plus on se trouve dans une situation où il existe une forte qualité institutionnelle et moins il est coûteux d'avoir recours à des contrats pour encadrer les transactions. Les acteurs ont alors intérêt à choisir ce mode de gouvernance plutôt que l'intégration verticale, si toutefois cette forme n'est pas imposée par les caractéristiques des transactions (Saussier et Yvrande-Billon [2007]). A l'inverse, plus on se trouve dans une situation d'aléas institutionnels, plus forte est 
l'incertitude et plus il est coûteux d'avoir recours aux contrats. Les travaux empiriques qui se sont intéressés à cette problématique portent généralement sur les décisions organisationnelles des firmes multinationales. Quand il s'agit de s'implanter à l'étranger, le mode de gouvernance choisi dépend ainsi non seulement des caractéristiques des transactions, mais aussi de la qualité des institutions dans le pays d'accueil. A titre d'exemple, Oxley [1999] montre que les entreprises américaines qui passent des accords de transfert de technologie avec l'étranger choisissent le mode de gouvernance de ces accords non seulement en fonction du type de technologie transférée, mais également du régime de protection des droits de propriété intellectuelle en vigueur dans le pays de l'entreprise partenaire. Dans les pays où ces droits sont mal protégés, les entreprises préfèrent des structures de gouvernance plus hiérarchiques (Figure 5).

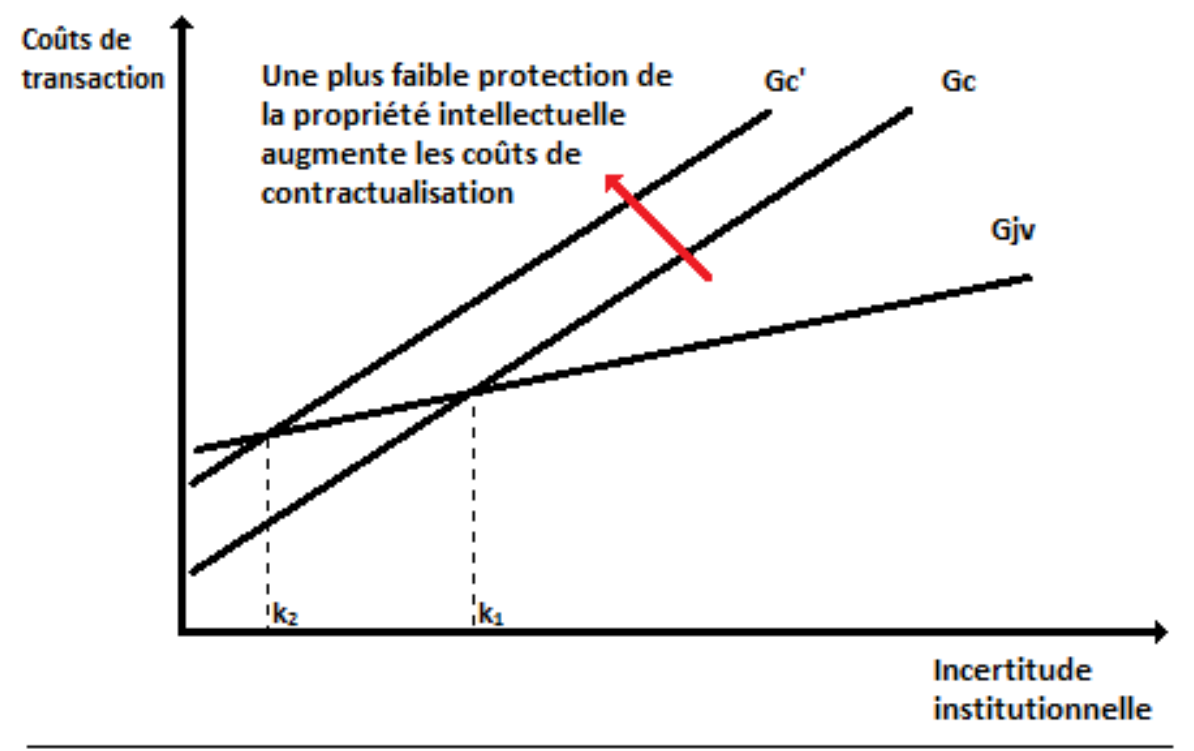

Figure 5 - Modification de l'arbitrage entre arrangement contractuel et coentreprise (joint venture) (adapté de Oxley [1999]). Les coûts de transaction liés à l'incertitude institutionnelle des arrangements contractuels augmente alors que la protection de la propriété intellectuelle diminue (Gc vers $\mathrm{Gc}^{\prime}$ ). Le point d'arbitrage entre la contractualisation et le recours au joint venture (Gjv) se déplace alors de $k_{1}$ vers $k_{2}$.

Deuxièmement, d'autres travaux s'intéressent à la capacité des agents économiques à s'adapter aux contraintes institutionnelles. Si l'environnement institutionnel limite le champs des modes de gouvernance possibles et interdit aux agents d'adopter le mode de gouvernance qu'ils jugent le plus efficient pour leurs transactions, on peut prédire que ceuxci chercheront à s'adapter aux contraintes de deux façons:

- en développant des mécanismes de coordination informels qui se substituent aux mécanismes formels dans le cas où ces derniers seraient éventuellement interdits par la réglementation. A titre d'exemple, les travaux de Palay [1984] sur le secteur ferroviaire de marchandise aux Etats-Unis, montrent que l'interdiction réglementaire de recourir à l'intégration verticale pousse les affréteurs et les transporteurs à recourir à des arrangements informels pour protéger leurs investissements spécifiques. 
- en modifiant les caractéristiques des transactions et en particulier le degré de spécificité des actifs de manière à aligner les attributs des transactions et le mode de gouvernance. L'article de Yvrande-Billon et Ménard [2005] montre ainsi que les opérateurs britanniques de transport ferroviaire de passagers s'adaptent à une réglementation qui les oblige à avoir recours à des contrats de courte durée en standardisant le matériel roulant, c'est à dire en réduisant le degré de spécificité de leurs actifs.

\section{Références}

ARROW K. J. et DEBREU G. [1954], "Existence of an Equilibrium for a Competitive Economy ", Econometrica, Vol. 22, $\mathrm{n}^{\circ} 3,265-290$.

BALMFORD A. et BOND W. [2005], "Trends in the state of nature and their implications for human well-being ", Ecology Letters, Vol. 8, n 11, 1218-1234.

BARKER T., KRAM T., OBERTHÜR S. et VOOGT M. [2001], "The Role of EU Internal Policies in Implementing Greenhouse Gas Mitigation Options to Achieve Kyoto Targets ». International Environmental Agreements, Vol. 1, $\mathrm{n}^{\circ} 2$ 2, 243-265.

BAYON R. [2004], " Making Environmental Markets Work: Lessons from Early Experience with Sulfur, Carbon, Wetlands, and Other Related Markets ", Forest Trends, Katoomba Group Meeting in Locarno, Switzerland, 2003.

BEKESSY S. A., WINTLE B. A., LINDENMAYER D. B., MCCARTHY M. A., COLYVAN M., BURGMAN M. A. et POSSINGHAM H. P. [2010], "The biodiversity bank cannot be a lending bank", Conservation Letters, Vol. 3, 151-158.

BROWN S. C. et VENEMAN P. L. M. [2001], " Effectiveness of compensatory wetland mitigation in Massachussetts, USA ", Wetlands, Vol. 21, n 4, 508-518.

BRUGGEMAN D. J., JONES M. L., LUPI F. et SCRIBNER K. T. [2005], "Landscape equivalency analysis: Methodology for estimating spatially explicit biodiversity credits ", Environmental Management, Vol. 36, 518-534.

BUTCHART S. H. M., WALPOLE M., COLLEN B., van STRIEN A., SCHARLEMANN J. P. W., ALMOND R. E. A., BAILLIE J. E. M. et al. [2010], "Global Biodiversity: Indicators of Recent Declines ", Science, Vol. 328, $\mathrm{n}^{\circ}$ 5982, $1164-1168$.

CDB [2011], "Conference of the Parties Decision X/2: Strategic plan for biodiversity 2011-2020 ", http://www.cbd.int/decision/cop/?id=12268.

CHABRAN, F. et NAPOLEONE C. [2013], " Les conditions du développement des banques d'actifs naturels en France ", Développement durable et territoires, Vol. 3, $n^{\circ} 1$.

CLEWELL A. F. et ARONSON J. [2010], La restauration écologique: Principes, valeurs et structure d'une profession émergente, Actes Sud.

COASE R. H. [1937], "The Nature of the Firm », Economica, Vol. 4, n 16, 386-405.

COASE R. H. [1960], "The Problem of Social Cost ", Journal of Law and Economics, Vol. 3, 1-44.

COASE R. H. [2000], "The Acquisition of Fisher Body By General Motors ", The Journal of Law and Economics, Vol. 43, $n^{\circ} 1,15-32$.

COGGAN A., BUITELAAR E., WHITTEN S. M. et BENNETT J. [2013], " Factors that influence transaction costs in development offsets: Who bears what and why? ", Ecological Economics, Vol. 88, 222-231.

COGGAN A., WHITTEN S. M. et BENNETT J. [2010], "Influences of transaction costs in environmental policy ", Ecological Economics, Vol. 69, n 9, 1777-1784.

DENISOFF C. et URBAN D. [2012], "Evaluating the Success of Wetland Mitigation Banks ", National Wetlands Newsletter. 
ETCHART G. [1995], "Mitigation banks: A strategy for sustainable development ", Coastal Management, Vol. 23, $\mathrm{n}^{\circ} 3,223-237$.

FALCONER K., DUPRAZ P. et WHITBY M. [2001], " An Investigation of Policy Administrative Costs Using Panel Data for the English Environmentally Sensitive Areas ", Journal of Agricultural Economics, Vol. 52, $\mathrm{n}^{\circ} 1,83-103$.

FLORES N. E. et THACHER J. [2002], " Money, Who Needs It? Natural Resource Damage Assessment ", Contemporary Economic Policy, Vol. 20, $\mathrm{n}^{\circ} 2,171-178$.

FOLKE C., CARPENTER S., WALKER B., SCHEFFER M., ELMQVIST T., GUNDERSON L. et HOLLING C. S. [2004], "Regime Shifts, Resilience, and Biodiversity in Ecosystem Management ", Annual Review of Ecology, Evolution, and Systematics, Vol. 35, 557-581.

GARRICK D., WHITTEN S. M. et COGGAN A. [2013], "Understanding the evolution and performance of water markets and allocation policy: A transaction costs analysis framework », Ecological Economics, Vol. 88, 195-205.

GIBBONS P. et LINDENMAYER D. B. [2007], "Offsets for land clearing: No net loss or the tail wagging the dog? " Ecological Management \& Restoration, Vol. 8, n 1, 26-31.

GOVERNMENT ACCOUNTABILITY OFFICE [2005], Wetlands Protection. Corps of Engineers Does Not Have an Effective Oversight Approach to Ensure That Compensatory Mitigation Is Occurring, Government Accountability Office.

GUSTAFSSON, B. [1998], "Scope and limits of the market mechanism in environmental management ", Ecological Economics, Vol. 24, n 2-3, 259-274.

HANSKI I. [1998], « Metapopulation dynamics », Nature, Vol. 396, n 6706, 41-49.

HARDIN, G. [1968], "The Tragedy of the Commons ", Science, Vol. 162, n 3859, 1243-1248.

HILDERBRAND R. H., WATTS A. C. et RANDLE A. M. [2005], "The Myths of Restoration Ecology ", Ecology And Society, Vol. 10, $\mathrm{n}^{\circ} 1,19$.

HOFFMANN M., HILTON-TAYLOR C., ANGULO A., BÖHM M., BROOKS T. M., BUTCHART S. H. M., CARPENTER K. E. et al. [2010], "The Impact of Conservation on the Status of the World's Vertebrates ", Science, Vol. 330, n 6010, 1503 -1509.

HOUGH P. et ROBERTSON M. [2008], "Mitigation under Section 404 of the Clean Water Act: where it comes from, what it means ", Wetlands Ecology and Management, Vol. 17, $\mathrm{n}^{\circ} 1,15$ 33.

ten KATE K., BISHOP J., et BAYON R. [2004], "Biodiversity offsets, views, experience and the business case ", IUCN, Gland, Switzerland and Cambridge, UK and Insight Investment, London, UK.

KENTULA M. E., SIFNEOS J. C., GOOD J. W., RYLKO M. et KUNZ K. [1992], « Trends and patterns in section 404 permitting requiring compensatory mitigation in Oregon and Washington, USA », Environmental Management, Vol. 16, $n^{\circ} 1$ 1, 109-119.

KING D. M. et HERBERT L. W. [1997], "The fungibility of wetlands ", National Wetlands Newsletter, Vol. 19, 10-13.

KLEIN B., CRAWFORD R. G. et ALCHIAN A. A. [1978], "Vertical Integration, Appropriable Rents, and the Competitive Contracting Process ", Journal of Law and Economics, Vol. 21, $\mathrm{n}^{\circ} 2$, 297-326.

KROEGER T. et CASEY F. [2007], "An assessment of market-based approaches to providing ecosystem services on agricultural lands ", Ecological Economics, Vol. 64, $n^{\circ} 2,321-332$.

LEVREL H. [2012], " Les acteurs économiques de la biodiversité. Exemple de l'impact des banques de compensation aux Etats-Unis ", Paru dans Biodiversité et contrat social : la réconciliation, 183-194, Editions Fayard, Paris. 
LEVREL H., PIOCH S., SPIELER R. [2012a], " Compensatory mitigation in marine ecosystems: which indicators for assessing the "no net loss" goal of ecosystem services and ecological functions? ", Marine Policy, Vol.36, 1202-1210.

LEVREL H., HAY J., BAS A., GASTINEAU P. et PIOCH S. [2012b], « Coût d'opportunité versus coût du maintien des potentialités écologiques: deux indicateurs économiques pour mesurer les coûts de l'érosion de la biodiversité ", Natures Sciences Sociétés, Vol. 20, 16-29.

LIBECAP G. D. [2011], "Institutional Path Dependence in Climate Adaptation: Coman's Some Unsettled Problems of Irrigation ", The American Economic Review, Vol. 101, n 1, 64-80.

MADSEN B., CARROL N., KANDY D. et BENNETT G. [2011], " 2011 Update. State of Biodiversity Markets Report: Offset and Compensation Programs Worldwide ", Ecosystem Marketplace.

MÄLER K., ANIYAR S., et JANSSON Å. [2008], "Accounting for ecosystem services as a way to understand the requirements for sustainable development ", Proceedings of the National Academy of Sciences, Vol. 105, n²8, 9501 -9506.

MARSH L. L. et ACKER D. R. [1992], " Mitigation banking on a wider plane ", National Wetlands Newsletter, Vol. 14, 8-9.

MARSHALL G. R. [2013], "Transaction costs, collective action and adaptation in managing complex social-ecological systems ", Ecological Economics, Vol. 88, 185-194.

MASTEN S. E., MEEHAN J. W. et SNYDER E. A. [1991], "The Costs of Organization ", Journal of Law, Economics, \& Organization, Vol. 7, $\mathrm{n}^{\circ}$ 1, 1-25.

MCCANN L. [2013], "Transaction costs and environmental policy design », Ecological Economics, Vol. 88, 253-262.

MCCANN L., COLBY B., EASTER K. W., KASTERINE A. et KUPERAN K.V. [2005], " Transaction cost measurement for evaluating environmental policies ", Ecological Economics, Vol. 52, $\mathrm{n}^{\circ} 4$, 527-542.

MCKENNEY B. A., et KIESECKER J. M. [2009], " Policy Development for Biodiversity Offsets: A Review of Offset Frameworks », Environmental Management, Vol. 45, n 1, 165-176.

MEA, Millennium Ecosystem Assessment [2005], Ecosystems and human well-being: synthesis, Washington D.C., Island Press.

MENARD C. [2000], « Une nouvelle approche de l'agro-alimentaire: l'économie néoinstitutionnelle ", Économie rurale, Vol 255, n 1, 186-196.

MENARD C. [2003], "Économie néo-institutionnelle et politique de la concurrence les cas des formes organisationnelles hybrides ", Économie rurale, Vol. 277, $n^{\circ}$ 1, 45-60.

MENARD C. [2004], "The Economics of Hybrid Organizations", Journal of Institutional and Theoretical Economics JITE, Vol. 160, n³, 345-376.

MÉNARD C. [2012], L'Economie des Organisations, Repères, La Découverte, Paris.

MENARD C. et SHIRLEY M. M. [2005], Handbook of New Institutional Economics, Springer.

METTEPENNINGEN E., BECKMANN V. et EGGERS J. [2011], "Public transaction costs of agrienvironmental schemes and their determinants-Analysing stakeholders' involvement and perceptions ", Ecological Economics, Vol. 70, n 4, 641-650.

MITSCH W. J. et GOSSELINK J. G. [2000], "The value of wetlands: importance of scale and landscape setting ", Ecological Economics, Vol. 35, $\mathrm{n}^{\circ}$ 1, 25-33.

MOILANEN A., van TEEFFELEN A. J. A., BEN-HAIM Y. et FERRIER S. [2009], " How Much Compensation is Enough? A Framework for Incorporating Uncertainty and Time Discounting When Calculating Offset Ratios for Impacted Habitat ", Restoration Ecology, Vol. 17, $\mathrm{n}^{\circ} 4$, 470-478.

MORENO-MATEOS D., POWER M. E., COMIN F. A., et YOCKTENG R. [2012], " Structural and functional loss in restored wetland ecosystems ", PLoS biology, Vol. 10, $n^{\circ} 1$. 
MORRIS R. K. A., ALONSO I., JEFFERSON R. G. et KIRBY K. J. [2006], "The creation of compensatory habitat-Can it secure sustainable development?", Journal for Nature Conservation, Vol. 14, $\mathrm{n}^{\circ} 2,106-116$.

NATIONAL RESEARCH COUNCIL [2001], Compensating for wetland losses under the Clean Water Act, National Academy Press, Washington DC.

NILSSON F. O. L. [2009], "Transaction costs and agri-environmental policy measures: are preferences influencing policy implementation? ", Journal of Environmental Planning and Management, Vol. 52, $n^{\circ}$ 6, 757-775.

NORTH D. [1991], "Institutions ", Journal of Economic Perspectives 5, n 1, 97-112.

OXLEY J. E. [1999], "Institutional environment and the mechanisms of governance: the impact of intellectual property protection on the structure of inter-firm alliances ", Journal of Economic Behavior \& Organization, Vol. 38, $\mathrm{n}^{\circ} 3,283-309$.

PALAY T. M. [1984], "Comparative Institutional Economics: The Governance of Rail Freight Contracting ", The Journal of Legal Studies, Vol. 13, n² 2, 265-287.

PANNELL D. J., ROBERTS A. M., PARK G. et ALEXANDER J. [2013] « Improving environmental decisions: A transaction-costs story ", Ecological Economics, Vol. 88, 244-252.

QUÉTIER F. et LAVOREL S. [2011], "Assessing ecological equivalence in biodiversity offset schemes: Key issues and solutions ", Biological Conservation, Vol. 144, n 12, 2991-2999.

RAMSEY F. P. [1928], " A Mathematical Theory of Saving ", The Economic Journal, Vol. 38, n 152, 543-559.

REES W. E. [1995], " Cumulative environmental assessment and global change ", Environmental Impact Assessment Review, Vol. 15, n 4, 295-309.

ROBERTSON M. [2004], " The neoliberalization of ecosystem services: wetland mitigation banking and problems in environmental governance ", Geoforum, Vol. 35, n 3, 361-373.

ROBERTSON M. et HAYDEN N. [2008], "Evaluation of a Market in Wetland Credits: Entrepreneurial Wetland Banking in Chicago ", Conservation Biology, Vol. 22, n 3, 636-646.

ROBERTSON, M. [2008], "The work of wetland credit markets: two cases in entrepreneurial wetland banking ", Wetlands Ecology and Management, Vol. 17, $\mathrm{n}^{\circ} 1$, 35-51.

RØRSTAD P. K., VATN A. et KVAKKESTAD V. [2007], "Why Do Transaction Costs of Agricultural Policies Vary? ", Agricultural Economics, Vol. 36, n 1, 1-11.

RUHL J. B. et SALZMAN J. [2006], "The Effects of Wetland Mitigation Banking on People ", National Wetlands Newsletter, Vol. 28, $\mathrm{n}^{\circ} 2,8-13$.

RUSSI D., ten BRINK P., FARMER A., BADURA T., COATES D., FÖRSTER J., KUMAR R., et DAVIDSON N. [2012], " The Economics of Ecosystems and Biodiversity for Water and Wetlands », Final Consultation Draft, 2012.

SALA, O. E., CHAPIN F. S., ARMESTO J. J., BERLOW E., BLOOMFIELD J., DIRZO R., HUBER-SANWALD E. et al. [2000], "Global Biodiversity Scenarios for the Year 2100 ", Science, Vol. 287, $n^{\circ}$ $5459,1770-1774$.

SALZMAN J. et RUHL J. B. [2002], " Paying to protect watershed services: wetland banking in the United States ", In PAGIOLA S., BISHOP P. S., LANDELL-MILLS N. (eds.), Selling forest environmental services: market-based mechanisms for conservation and development, 1-14, Earthscan, London.

SALZMAN J. et RUHL J. B. [2000], "Currencies and the Commodification of Environmental Law ", Stanford Law Review, Vol. 53, $\mathrm{n}^{\circ} 3,607$.

SAUSSIER S. [1997], "Choix contractuels et coûts de transaction : une analyse économique des contrats d'approvisionnement en charbon d'EDF ", Thèse de doctorat, Université Paris 1 Panthéon Sorbonne. 
SAUSSIER S. et YVRANDE-BILLON A. [2007], Économie des coûts de transaction, Paris, Découverte. SCEMAMA P. et LEVREL H. [2012], "La méthode HEA: cadre d'application, fonctionnement et exemple ", Rapport Onema.

SCRIBNER K. T., BLANCHONG J. A., BRUGGEMAN D. J. et al. [2005], " Geographical Genetics: Conceptual Foundations And Empirical Applications Of Spatial Genetic Data In Wildlife Management ", Journal of Wildlife Management, Vol. 69, $n^{\circ}$ 4, 1434-1453.

SIMON H. A. [1961], Administrative behavior; a study of decision-making processes in administrative organization, New York, Macmillan.

SPILLER P. T. et TOMMASI M. [2005], "The Institutions of Regulation: An Application to Public Utilities ", In MENARD C. and SHIRLEY M. (eds.), Handbook of New Institutional Economics, 515-543, Springer, Netherlands.

STAVINS R. N. [1998], « What Can We Learn from the Grand Policy Experiment? Lessons from SO2 Allowance Trading ", Journal of Economic Perspectives, Vol. 12, n 3, 69-88.

STEELE, S. R. [2009], "Expanding the solution set: Organizational economics and agrienvironmental policy ", Ecological Economics, Vol. 69, n² 2, 398-405.

THÉBAUD, O., INNES, J. et ELLIS N. [2012], "From anecdotes to scientific evidence? A review of recent literature on catch share systems in marine fisheries ", Frontiers in Ecology and the Environment, Vol. 10, $\mathrm{n}^{\circ}$ 8, 433-437.

UICN [2009], Wildlife in a Changing World - An Analysis of the 2008 IUCN Red List of Threatened Species, Gland, Switzerland. UICN. VIÉ J. C., HILTON T. C. et STUART S.N.

WALKER S., BROWER A. L., STEPHENS R.T. T. et LEE W. G. [2009] « Why bartering biodiversity fails ", Conservation Letters, Vol. 2, $n^{\circ} 4,149-157$.

WILLIAMSON O. E. [1985], The economic institutions of capitalism : firms, markets, relational contracting, New York, London, Free Press, Collier Macmillan.

WILLIAMSON O. E. [1991], "Comparative Economic Organization: The Analysis of Discrete Structural Alternatives ", Administrative Science Quarterly, Vol. 36, n² 2, 269-296.

WILLIAMSON O. E. [1993], "Opportunism and Its Critics", Managerial and Decision Economics, Vol. 14, n², 97-107.

WILLIAMSON O. E. [1996], The Mechanisms of Governance, Oxford University Press.

YVRANDE-BILLON A. et MENARD C. [2005], " Institutional constraints and organizational changes: the case of the British rail reform », Journal of Economic Behavior \& Organization, Vol. 56, $\mathrm{n}^{\circ}$ 4, 675-699.

ZAFONTE M. et HAMPTON S. [2007], "Exploring welfare implications of resource equivalency analysis in natural resource damage assessments ", Ecological Economics, Vol. 61, $\mathrm{n}^{\circ} 1,134-$ 145.

ZEDLER J. B. et CALLAWAY J. C. [1999], « Tracking Wetland Restoration: Do Mitigation Sites Follow Desired Trajectories? ", Restoration Ecology, Vol. 7, n 1, 69-73. 